\title{
Geleitwort
}

\section{des Präsidenten der Akademie der Wissenschaften zu Göttingen}

Carl Friedrich Gauß, seit seinem 30. Lebensjahr bis zu seinem Tode im Jahre 1855 Professor an der Georgia Augusta und zugleich Ordentliches Mitglied der Königlichen Societät der Wissenschaften zu Göttingen, begann als Mathematiker. Er galt schon zu seinen Lebzeiten und gilt heute noch als princeps mathematicorum. Er entwickelte Methoden, mit denen die Bahn des neuentdeckten und wieder aus dem Blick geratenen Planeten Ceres berechnet werden konnte, was zu dessen Wiederauffindung führte. Dies brachte ihm die Göttinger Professur für Astronomie ein. Hier gab er der theoretischen Astronomie eine neue Grundlage. In Göttingen beschäftigte sich Gauß auch mit der Geodäsie und praktizierte sie bei der Vermessung des Königreichs Hannover. Angeregt durch die Führung der Professorenwitwen- und -waisenkasse, schuf er die Grundlagen der Versicherungsmathematik. In seiner physikalischen Periode beschäftigte er sich unter anderem mit dem Erdmagnetfeld und dem Elektromagnetismus. Damit legte er die Grundlagen für die elektromagnetische Telegraphie.

Gauß unterhielt seit Beginn seiner wissenschaftlichen Karriere vielfältige wissenschaftliche Beziehungen zu Russland, die in dem vorliegenden Band erstmals umfassend dokumentiert werden. Im Zentrum des Bandes steht die 1725 gegründete Kaiserliche Akademie der Wissenschaften in Sankt Petersburg, zu deren Korrespondierendem Mitglied Gauß bereits 1802 gewählt wurde, d.h. noch bevor er zunächst als Korrespondierendes, einige Jahre später als Ordentliches Mitglied in die Societät der Wissenschaften zu Göttingen aufgenommen wurde. 13 der 17 Korrespondenten von Gauß waren Mitglieder der Petersburger Akademie. Diese war damals die bedeutendste Wissenschaftsinstitution Russlands. Der Inhalt der Korrespondenz berührt alle Forschungsgebiete von Gauß. So kann der Leser des Buches anhand des Briefwechsels Gauß' Einfluss auf zahlreiche in Russland wirkende Wissenschaftler im Einzelnen nachvollziehen. Der Band dokumentiert den großen Einfluss, den die Akademien zu Beginn des 19. Jahrhunderts auf die Entwicklung der Wissenschaften und den dazu gehörenden Austausch unter den Akademiemitgliedern ausübten. Im ersten Teil wird eine Übersicht über den Stand der Wissenschaften in Russland zu Lebzeiten von Gauß gegeben. Am Schluss des ersten Teils werden die Übersetzungen des Werkes von Gauß ins Russische behandelt und eine komplette Liste der Übersetzungen vorgelegt. Der gesamte Band ist von dem Vizepräsidenten der Göttinger Akademie, dem Slavisten Werner Lehfeldt, 
redigiert worden, der zu ihm auch einen Abschnitt über die Beschäftigung von Carl Friedrich Gauß mit der russischen Sprache beigesteuert hat.

Möge das hiermit vorgelegte Werk zur Wissenschaftsgeschichte den gegenwärtigen Austausch unter Wissenschaftlern, besonders die wissenschaftlichen Beziehungen zwischen Deutschland und Russland, fördern und die Bedeutung der Akademien der Wissenschaften für die Entwicklung der Wissenschaft ins Licht rücken.

Christian Starck 